\title{
Apuntes para una histarla de la Facultad de Educación de la Universidad Surcolombiana 1
}

\author{
Wisberto Navarto Salcedo \\ Docente Programa de Psicopedagogia \\ wisberto@usco.edu.co \\ Miguel Angel Mahecha \\ Docente Programa de Lengua Castellana \\ mamahechab@usco.edu.co \\ Facultad de Educación
}

Preámbulo

Con ocasión de la celebración de los treinta y tres años de la Facultad de Educación, hemos decidido reunir datos, recuerdos y reflexiones sobre una instancia académica-administrativa que tanto le ha aportado a la constitución interna de la Universidad Surcolombiana como a la región en donde se desarrolla. Es claro que estos apuntes no aspiran a ser un tratado histórico; su pretensión es más bien aportar referencias puntuales de cada uno de los momentos que ha vivido la Facultad.

No obstante, es pertinente mencionar que este trabajo presenta la primera parte de la reconstitución de la historia de la Facultad, tomando inicialmente como punto teórico de referencia, lo propuesto por Mankeliunas (1993,43-66), "la forma cronológica presenta la sucesión de eventos sobresalientes en el desarrollo de una ciencia, buscando nexos causales; de ordinario el tiempo se divide en

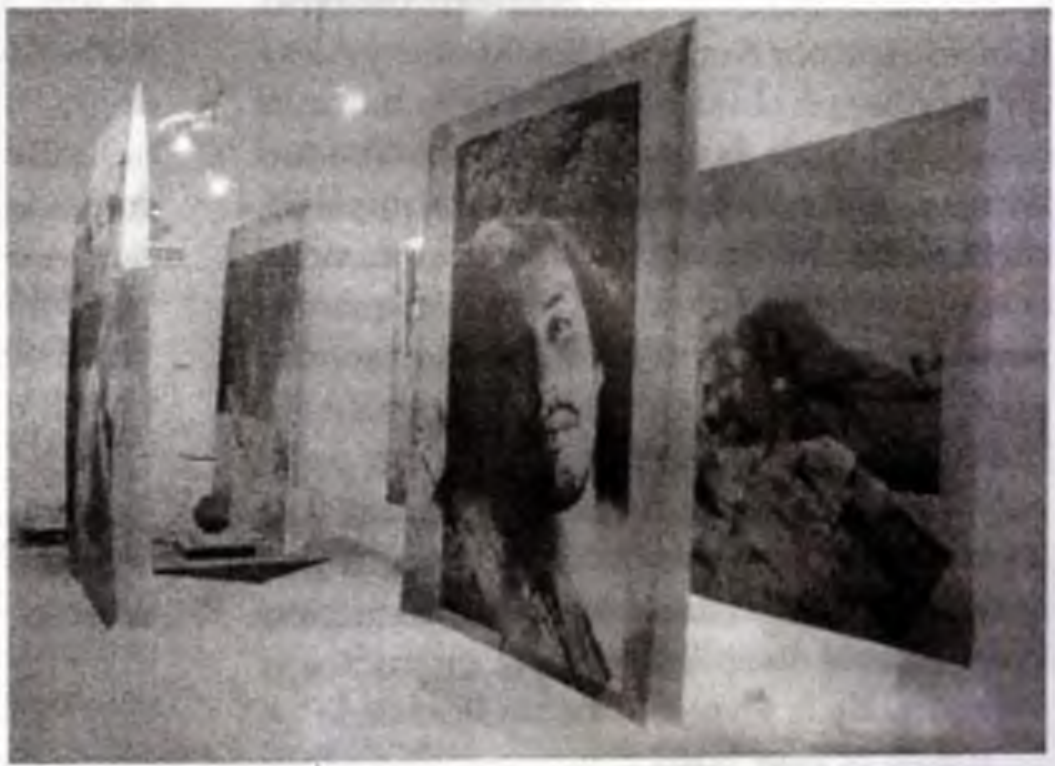

JADER RIVERA: "SUDARIOS" INSTALACIÓN FOTOGRAFICA 2009 -Teruel

'Estos: apuntes hacen parte del Proyecto de Investigacion del estado del Arte de la Pedagogia en la Facultad 
saberes disciplinar y pedagógico. Al citar nombres, articulos y libros producidos, eventos realizados, logros y por qué no frustraciones, se está llevando a cabo un procedimiento cronológico/autobiográfico. Queda en el aire, el tema de una historia de la Facultad por problemas; seguimos con Mankeliunas, "El estudio del desarrollo de los problemas, de su aparición en el contexto de las ideas de época, y su debate o su negación, aclara mejor los mismos problemas existentes en la actualidad y proporciona luces para su solución. Es decir, además del valor científico tiene valor didáctico especialmente para los principiantes. Este camino ayudará a comprender mejor tanto el desarrollo conceptual como epistemológico." Esto quiere decir que se analizarán en una etapa ulterior, los trabajos académicos (i.e. investigativos) de los docentes adscritos a la Facultad de Educación para verificar hasta qué punto han integrado de manera explícita: saber disciplinar y saber pedagógico.

Hemos decidido incluir en primer lugar, a manera de exordio, algunas de las reflexiones pedagógicas propuestas en el documento Modelo Curricular para la Formación Integral Estructurado por Núcleos, Dimensiones y Areas redactado en el año de 1999 bajo la coordinación de la profesora Helga Truque Guzmán quien lideró un valioso equipo de colaboradores ${ }^{2}$. Los datos que se presentan enseguida son el fruto de un cuestionamiento permanente por parte de los Decanos y los profesores de la Facultad en torno a la "formación de docentes" y su impacto en la región surcolombiana. Siendo más específicos, la preocupación siempre ha sido el cómo lograr de manera equilibrada una adecuada formación en los saberes disciplinar y pedagógico que son los pilares de la identidad de un licenciado. A más de esto se ha buscado igualmente esbozar los campos de acción, las problemáticas más recurrentes y los modelos teóricos más acertados para comprender el plano pedagógico como parte integral de un sistema educativo. Es por eso que leemos en el documento citado:

Este enfoque sistémico que orienta el Modelo Curricular para la formación docente de la Facultad de Educación de la Universidad Surcolombiana, parte de considerar que la pedagogia no puede reducirse a una reflexión sobre la enseñanza como uno de los componentes de la didáctica, sino que fundamenta el análisis pedagógico en la articulación de lo macro y lo micro en los social y lo cultural. Además, al considerar lo pedagógico, como interacción, se resalta la importancia del diálogo, la interacción y reflexión del maestro sobre su práctica pedagógica, entendida como la aplicación de conocimientos teóricos y metodológicos en la dinamización de procesos educativos, concebidos como procesos de interacción humana orientados por intencionalidades formativas $y$ de cualificación permanente.(página 7)

No hay que olvidar que el 'proyecto pedagógico' de la Facultad de Educación está en (re)constitución y no puede dejar de lado sus antecedentes.

La Pedagogía es el saber especifico de las Facultades de Educación, unidades académicas de las Universidades encargadas de formar integral e integramente a los licenciados.

El proceso educativo como se sabe, ha sido objeto de reflexión desde la antigüedad, pero la pedagogía como tal sólo surgió al ritmo del desarrollo científico-técnico, permeada por las

\footnotetext{
Fueron los profesores Guillerno Sánchez Amaya, Alba Luz Quintero Charry, Ana Lucinda Pachón Maldonado, David Augusto Plata Ramirez, Fabiola Gälvez Toro, Jaito Ramirez Bahamón, Leonel Arias Guriérrez, Luz Stella Gonzälez Salamanca, Margarita Chacoin Trujillo, Miller Dussán Calderón, Myrian Oviedo Córdoba, Nohora Elena Rujns, Ofelia Ranirez Losada y Wisberro Navarro Salcedo.
} 
preocupaciones hacia la naturaleza y por las formas de la racionalidad ilustrada. De ahi que su origen estuviera emparentado con una concepción positiva, objetiva y racional y sus investigaciones orientadas en los modelos de las ciencias naturales y afines, siendo su naturaleza más socio humanistico como lo demostraron sus propias crisis.

"... a finales del siglo XIX y comienzos del $\mathrm{XX}$, cuando aquellos modelos (los positivistas) entraban en crisis, la pedagogía encontró sus nuevos fundamentos en las ciencias sociales, en la filosofia de la historia y en las ciencias del espiritu."

De manera que si en el pasado los problemas de la enseñanza eran de fácil solución desde los modelos de la naturaleza, en los últimos tiempos, la pedagogia y la educación, como campos de las ciencias sociales, son entendidas como complejas y vienen a compartir múltiples significaciones, muy propias por cierto, de toda disciplina que aspire a la cientificidad.

De lo anterior se deduce entonces que la pedagogia contemporánea tiene una doble raiz, la que se ubica en su procedencia racionalnatural, que la asume como teorias hechas de conceptos, nociones racionales, leyes universales y análisis experimentales, y otra que la entiende como "pensamientos complejos, experiencias enigmáticas, producto de la irrupción del azar y lo problemático en la vida de las cosas, de los individuos, de la sociedad. Por estar la pedagogía inmersa en el campo de lo complejo, no ha sido fácil precisar su carácter disciplinar como saber o como ciencia autónoma. Muchos estudios tienden a reconocerla como una disciplina aún bajo diferentes denominaciones y teorias.

Hay quienes la han concebido como una de las disciplinas que conforman las "ciencias de la educación" y la reducen a un conjunto de técnicas, procedimientos y usos de medios auxiliares para la enseñanza, confundiéndola con la estrategia didáctica.

Otros le han asignado el papel de disciplina sintetizadora, como un híbrido de psicologia, sociología, economía, etc., sin llegar a identificar en ella un objeto específico.

"Una tercera mirada la asume como la explicación sobre lo educativo, es decir, sobre los fines que persigue la educación, los lugares que se asignan a los agentes que en ella intervienen, la fragmentación y enunciación que sufre el saber al ser sometido a los procesos de institucionalización, las formas de interacción que se establecen entre los sujetos, la normatividad que formaliza esas relaciones y la apropiación que un sistema social permite frente a la ciencia, la técnica y la cultura"

Quienes defienden esta tercera visión, consideran además, que la pedagogia, al ser una explicación del hecho educativo y al ser éste un hecho social, sólo puede llegar a ser comprendida en su movimiento histórico.

Para algunos epistemólogos, la legitimidad de estos tres enfoques de cara al enriquecimiento de las posibilidades de opción del maestro concreto, es a todas luces evidente. Pero mantienen el criterio de que la pedagogia aún continúa caracterizada por la ambigüedad de sus propuestas y por la relativización de sus saberes y que por tanto, no puede alcanzar el estatuto cientifico que identifica el ideal del conocimiento moderno; sin embargo otros autores sostienen que la pedagogía puede construir sus estatutos científicos, interrelacionados con otras ciencias.

"La pedagogia puede hacer valer su estatus cientifico y lo acertado de su pretensión en tanto que ciencia social, en la medida en que se apoye en ciertas ciencias 
humanas y sociales básicas como la psicología, la sociologia y la lingüistica, sin que tenga que reducirse a éstas, ni perder su autonomía epistemológica por ello."

Por su parte, el profesor Flórez, encuentra una unidad teórica en la pretensión de la pedagogía en convertirse en ciencia, aunque admite que ha existido cierta ambigüedad epistemológica en la pedagogía originada en la historicidad interna de su objeto, en el carácter interrumpido de la cultura, en la inestabilidad del contexto propio del aprendiz..."

... avanza en su obra (Hacia una pedagogía del conocimiento) en la formulación de algunos principios, criterios y "sentidos" a los que según él, han respondido y responden "todas las teorias pedagógicas desde Comenio hasta nuestros dias "y que permiten vislumbrar cierta unidad teórica en el proceso de distinguir cual es el pensar y el hablar pedagógico de una comunidad intelectual.

Hoy por ejemplo, con mayor recurrencia los estudios aceptan caracterizar la pedagogía como una disciplina en construcción, siempre y cuando esto no conlleve ninguna connotación de "humildad" o "subdesarrollo", sino que signifique el reconocimiento de la alta inestabilidad histórica de la relación teoría-práctica, así como del carácter cambiante $y$ multivariado de los procesos educativos en cada sociedad, época, región o nivel educativo.

Este ser de la pedagogia, como un conocimiento en construcción se apoya en la corriente Foucoltiana, donde algunos autores se mueven hoy bajo la noción de saber pedagógico.

Se acoge la tesis que un saber no conduce necesariamente a la formación de una ciencia, pero que es indispensable para su formación; y que el saber es el espacio más amplio y abierto de un conocimiento, donde se pueden localizar discursos de muy diferentes niveles, desde aquellos que apenas empiezan a tener objetos de discursos y prácticas hasta los que "logren cierta sistematicidad, todavía no ligada a criterios formales"

Desde ésta visión, el saber pedagógico comprenderá, desde situaciones prácticas de la enseñanza, la escuela y el maestro, hasta las situaciones conceptuales de la pedagogía como la comunicación de la didáctica con los saberes enseñados y los problemas de la pedagogía en la interioridad de las ciencias de la educación.

También en los estudios actuales existe cierta unanimidad en el rechazo a la idea de considerar la pedagogía como una más entre las llamadas ciencias de la educación.

Se afirma que disciplinas como la sociologia, la lingüística y la psicologia, contribuyen a explicar el fenómeno educativo, cada una desde su especificidad conceptual y metodológica, tomando cada una aquel aspecto de la educación involucrando en su propio objeto, pero se afirma también que ellas no suplen competentemente el marco teórico experiencial de la pedagogía, a la que autores como Flórez no dudan el llamar "la ciencia de la educación por excelencia".

\section{PEDAGOGIA Y FACULTADES DE EDUCACIÓN}

Que la pedagogía tenga o no status científico o que permanezca en el umbral de la ambigüedad, parece no ser ya el de las discusiones en nuestras universidades.

Hoy la mayor preocupación es por la ausencia de una verdadera cultura pedagógica en las facultades de educación, al comprobar que en su seno sigue latente lo que desde comienzo de la actual década se llamó el "vaciamiento" del 
sentido de la pedagogia" al quedar ésta limitada a las prácticas instrumentalizadas de sus docentes y perder en consecuencia, toda connotación de los procesos históricos a lo largo de los cuales se construye el fundamento teórico que interactúa con esta práctica social.

"Estudios recientes confirman que las Facultades de Educación no han vislumbrado aún un camino para acceder a la pedagogia en sus diferentes versiones, que en ellos se ha reforzado una visión de currículo en donde las didácticas especiales subsisten sin un diálogo con la pedagogia y la didáctica general, desconociendo los aportes de la tradición pedagógica a la enseñanza y al aprendizaje.

En fin, que se manifiesta en ellas un doble desequilibrio: la formación superficial en la ciencia por enseñar y la formación superficial en pedagogia y didáctica"

El resultado de esta situación es que se viene formando un maestro fuera de un panorama conceptual que lo enmarque, que a ese maestro se le ha relegado a ser un especialista sin una visión adecuada sobre la complejidad de las situaciones que lo acechan que se le ha relegado igualmente a ser un maestro sin identidad con un saber $y$ un hacer que lo concretan como profesional y como intelectual y que fortaleza su imagen social.

Para superar este "vaciamiento", estudios, seminarios y foros educativos recientes han planteado con especial énfasis la necesidad de que las instituciones formadoras de docentes reivindiquen como su objeto fundamental lo pedagógico.

Además, las disposiciones de los Decretos 272 de 1998 y 2566 de 2003 constituyen un llamado a la comunidad universitaria a asumir lo pedagógico como espacio teórico y epistemológico para la producción, la investigación y la apropiación del conocimiento, un llamado también para que la pedagogia no siga siendo un asunto puramente estratégico e instrumental, sino que se la catalogue como la disciplina fundante que orienta y da sentido a la formación de formadores y a la actividad general de las facultades de educación.

La sociedad reclama licenciados con una formación sólida en los saberes especificos, en los núcleos del saber pedagógico a saber: la Educabilidad, la Enseñabilidad, la estructura histórica y epistemológica de la Pedagogia y las realidades y tendencias sociales y educativas institucionales, nacionales e internacionales en las dimensiones éticas, cultural y politica de la profesión educativa. Las instituciones educativas requieren educadores capaces de trabajar en equipos para conformar comunidad académica. Exigen profesionales capaces de crear nuevos modelos pedagógicos que superen la pedagogia normativa, vertical, memorista, libresca y descontextualizada. Se requieren educadores democráticos capaces de ofrecer a sus alumnos diferentes estrategias pedagógicas para el logro de las competencias que necesitan demostrar en cada área académica. Un educador autónomo capaz de permitir el ejercicio de la autonomia, la libertad de pensamiento y de la democracia participativa. En el ámbito de la investigación pedagógica se requiere adoptar e implementar las lineas propias de la Facultad y de cada programa académico en el plan de desarrollo investigativo. Otro tanto se espera hacer con el plan de extensión o servicio social para que las instituciones educativas se beneficien de las innovaciones que se generen en las facultades. No ha existido un modelo pedagógico que responda a la formación integral. Ha sido predominante el Racionalismo Académico, la Tecnología Educativa (Conductismo) y Prácticas de Pedagogia Activa, Pedagogía Cognitiva, Pedagogia Participativa y Prácticas Pedagógicas 
Empiristas.

El Racionalismo Académico surge en aporte a la profundidad conceptual por interpretar los procesos de desarrollo. Responde parcialmente a la solución de los problemas sociales e impuso el aprendizaje memoristico.

La Tecnologia Educativa implementó un enfoque administrativista $y$ de control instrumental de la conducta.

Los nuevos modelos pedagógicos de la Pedagogia Activa, Participativa y Constructivista fomentan la capacidad comprensiva pero su práctica es incipiente por lo cual no responden de manera adecuada al desarrollo científico, tecnológico, humanistico ni a las características de multiculturalidad, interculturalidad y biodiversidad de la región y del país.

En la Facultad de Educación de la Universidad Surcolombiana, no existe un consenso suficiente sobre un modelo pedagógico integral, ético y democrático, pero se está actuando en su dilucidación e implementación a partir de la interiorización de los principios y planteados en la teleologia institucional de la Facultad y de cada programa. Hoy, el modelo utilitarista y la racionalidad instrumental pernean todo el ambiente de la cotidianidad universitaria. La Facultad debe desarrollar el sistema de referentes axiológicos de la USCO: dignidad humana, autonomia, rigurosidad académica, democracia participativa, identidad regional y sostenibilidad del desarrollo.

Los modelos curriculares de la Universidad Surcolombiana han obedecido a imperativos legales emanados del Ministerio de Educación Nacional y del ICFES, más que a modelos pertinentes estudiados, debatidos y adoptados por la misma Universidad. El modelo curricular vigente es desagregado, organizado en áreas académicas y asignaturas.

El nuevo sistema curricular adoptado por la
Universidad tiene como eje la flexibilización y la estructuración de cada programa académico en dos componentes: El Componente Básico y el Componente Complementario Flexible que requieren una reconceptualización y reestructuración curricular de Fondo para lograr curriculos integrados, holísticos y dinámicos.

El diseño curricular se organiza en créditos académicos los cuales obedecen a la dedicación del estudiante y la labor del docente, abandonando el criterio economista.

La conversión del sistema anterior a créditos académicos parte de la definición de los Proyectos Educativos de los Programas PEP, los cuales son la base de los Planes de Mejoramiento de cada programa académico. Esta situación exigió un ajuste al plan de Desarrollo toda vez que los Planes Educativos de Programas PEP y los Planes de Mejoramiento PEM apuntan a lograr y sostener la calidad de la formación de los Licenciados.

A la Facultad de Educación le compete, al interior de la Universidad Surcolombiana liderar la participación en la construcción del Proyecto Educativo de la Universidad Surcolombiana PEU- el cual fue adoptado por acuerdo No 020 del 13 de mayo del 2003, emanado del Consejo Superior, en cuyo debate, formulación y adopción participaron profesores del Departamento de Psicopedagogia y de los programas de la Universidad. Además, debe dirigir y orientar el sistema curricular, adoptado por acuerdo $\mathrm{N}^{\circ} 018$ del 13 de mayo del 2003, emanado del Consejo Superior; capacitar a los profesores de la Universidad en Docencia Universitaria y en métodos pedagógicos actualizados; realizar investigación sobre los procesos pedagógicos internos y participar en los proyectos de desarrollo académico institucional. Según la Misión de la Universidad Surcolombiana acordada a través del PEU, la razón de ser institucional se centra en la 
"Formación integral de profesionales a través de la asimilación, producción, aplicación y difusión de conocimiento científico, humanistico, tecnológico, artístico y cultural, con espiritu critico, para que aborden eficazmente la solución de los problemas relevantes del desarrollo humano integral de la región Surcolombiana con proyección nacional e internacional, dentro de un marco de libertad de pensamiento, pluralismo ideológico y de conformidad con una ética que reivindique la solidaridad y la dignidad humana".

Y para el caso especifico de la Facultad de Educación su misión es "la formación de educadores con un sentido humanista e integral, en las diferentes áreas del conocimiento y la producción y consolidación de nuevos saberes y en lo esencial el pedagógico, para el mejoramiento de la educación como factor fundamental del desarrollo integral, equitativo y sostenible de la región Surcolombiana, el pais y la esfera internacional."

\section{Datos históricos}

La Facultad de Educación de la Universidad Surcolombiana fue creada por Acuerdo Número 034 del 15 de junio de 1977 emanado del Consejo Superior. Su primera decana fue la doctora Myriam Botero Jaramillo (periodo 1977 . 1980) quien logró consolidar la organización administrativa y académica de la Facultad. Siete profesores hicieron parte de esta etapa: Roberto Romero, Olga Tony Vidales, Leonel Arias, Luis Humberto Alvarado Edgar Machado, Jorge Guebelly y Misael García. Se dejó además un camino bien trazado para que el segundo decano, el doctor Nelsón López (periodo 1980. 1984) adelantara proyectos que fortalecian la identidad de la propia Facultad - logrando que se convirtiera en la segunda mejor facultad de educación del pais-, entre los que se cuentan la aprobación, por parte del Consejo Académico de la unificación del área de formación profesional docente para todas las carreras de la Facultad. Se creó el departamento de Psicopedagogía. Se propuso la estructura curricular del área de investigación la cual se acogió para todas las carreras de la Universidad; se le dio una atención especial a la práctica profesional docente así como a la práctica administrativa docente que ejecutan los programas de la Facultad logrando reglamentar y cualificar estos procesos.

En el seno del programa de lingüística y literatura se lleva a cabo una de las primeras experiencias investigativas de la Facultad a través del CESCO (Centro de Estudios Surcolombianos) en la comunidad indigena de Guambia, establecida en Silvia Cauca, que dejó la redacción de un diccionario bilingüe españolguambiano. En el año de 1976 el mismo programa realizó el estudio preliminar de El Atlas Lingüístico del Huila bajo la coordinación de los profesores Luis Humberto Alvarado y Rafael Cortés Murcia. La seriedad y relevancia del proyecto, merecieron no solamente el apoyo financiero de COLCIENCIAS con la suma de 261 mil pesos, de la Industria licorera del Huila, la Asamblea Departamental, el municipio de Aipe; sino también con la asesoría de importantes instituciones a nivel nacional e internacional. Lastimosamente son pocos los documentos en buen estado que logren dar testimonio de dicho proyecto y se encuentran deteriorándose en un mueble desvencijado de la universidad; ni siquiera queda un ejemplar en la biblioteca central.

Ahora bien, dos hechos significativos para el desarrollo de la Universidad se produjeron en la década de los ochenta: la adopción de la estructura orgánica acogida mediante Decreto Nacional 3092 de 1983 y la aprobación por parte del Consejo Superior del Primer Plan de Desarrollo de la Universidad. Estas fases de organización y planeación generaron al interior de la Facultad un ambiente de reflexión y toma de decisiones relacionadas con proyectos y planes impulsados durante los periodos administrativos 
de los decanos Jesús María Vidal (primer decano elegido por voto popular, periodo 1984-1988) y Jorge Elias Guebelly (periodo 1986-1988).

Las licenciaturas en Administración Educativa, Lingüistica y Literatura y Educación Preescolar fueron inicialmente tecnologías creadas por el antiguo ITUSCO. Al profesionalizar estas carreras se demostró que las tecnologias en el campo educativo no respondian a necesidades sociales ni a expectativas comunitarias ni estaban respaldadas por la política educativa vigente. Tampoco se pudo establecer una diferencia entre el nivel tecnológico y el nivel profesional por lo cual al pasar de 'tecnologia' a 'licenciatura' estos programas no lograron fundamentar suficientemente los nuevos currículos sino que se procedió a aumentar el número de asignaturas y a adecuar el plan de las exigencias legales del ICFES.

Esto explica por qué surge paralelamente en estos tres programas la necesidad de estructurar los planes de estudio para adecuarlos a nuevas exigencias pedagógicas y socioeducativas. De ahi que en el año de 1985 se señalaron como perspectivas de desarrollo de la Facultad la creación de la Licenciatura en Lenguas Modernas, el postgrado en Docencia Universitaria, el postgrado en filosofía, la creación de la carrera de Educación básica primaria, la investigación sobre el perfil profesional del docente en el Huila, asi como la capacitación en renovación curricular, la autoevaluación y la reestructuración de cada programa.

En el quinquenio $80-85$ se iniciaron las primeras acciones de integración interfacultades como el 'Modelo para capacitación e incorporación de los agentes de la medicina tradicional de salud en el tratamiento de la diarrea infantil' y la 'Evaluación de las destrezas y motivación de los estudiantes de primero y segundo año frente a la carrera de medicina'. Investigaciones que asesoró el área de investigación del departamento de Psicopedagogía como colaboración al programa de Medicina.

Desde la Facultad se administraron los servicios de docencia en Investigación, Matemáticas, Psicologia, Sociologia, Técnicas de comunicación, Idiomas además de la asesoría en actividades deportivas y recreativas a los demás programas de la Universidad.

También a partir de 1980 principalmente como conclusión de los estudios e investigaciones que adelantaron los programas de Lingüística y literatura, Administración Educativa y Educación Física, se vio la necesidad de fundamentar los programas con un marco histórico (geográfico, económico, educativo, político y cultural) para formular la propuesta de "Perfil Profesional Real" que permitiera articular la Facultad con el desarrollo regional. Desde entonces, viene la propuesta de, en palabras del profesor Jesús Maria Vidal, ruptura en la forma tradicional de circunscribir la extensión a los aspectos netamente colaterales a la acción docente de la práctica y convertir el desarrollo académico en una acción simultánea de docencia y extensión por parte de los profesores y estudiantes como una modificación metodológica en el mismo enfoque de las asignaturas que fue planteada inicialmente desde el programa de Lingüistica y literatura. ¿Cuál fue el resultado de esta proposición? El programa de Alfabetización, Postalfabetización y Desarrollo Comunitario no sólo a nivel rural sino también a nivel urbano cuyo liderazgo estuvo en manos del profesor Gustavo Briñez. Precisamente gracias a los trabajos con el proyecto de alfabetización llevado a cabo en El Pato, Balsillas y Rovira y financiado por el MEN, el profesor Briñez, quien estaba a la cabeza de dicho proyecto, recibió en 1990 , año consagrado a la alfabetización, la medalla SIMON BOLIVAR. 
Se realizó un estudio para la transformación del Jardín Infantil en IDAP (Instituto de Aplicación Pedagógica), se planteó además convertir el programa de Matemáticas y Fisica en Departamento de Ciencias Básicas con áreas especificas en Biología, Química, Matemáticas, Fisica, y la creación de un taller de diseño de materiales didácticos por el área de Física, la investigación en el campo de la energía solar y la organización de un centro de microprocesadores.

La propuesta más innovadora que se adoptó en este periodo que afectó, como ya se ha dicho, tan to la estructura académica, curricular, interna de la Facultad como el establecimiento de una nueva relación con los municipios e instituciones educativas, fue la creación de la Licenciatura en Tecnología Educativa ${ }^{3}$ (que ya no existe hoy). La Universidad Surcolombiana apoyó y adoptó el Modelo Surcolombiano para la Educación Superior Abierta y a Distancia y fue liderado por el profesor Rafael Cortés Murcia (cf. Acuerdos 03/83 emanado del Consejo Académico y $37 / 83$ del Consejo Superior.)

Los dias 20, 21 y 22 de octubre de 1983, la Facultad de Educación -en colaboración con el ICFES-, organizó el Taller Nacional de Práctica Profesional Docente. Los miembros del comité organizador fueron los profesores Nelson López, Carlos Bonilla y María de los Angeles Rivera. El propósito del taller fue analizar, reflexionar y evaluar el estado actual de la práctica docente en el país, con el fin de fijar políticas unificadas dirigidas a sistematizar criterios y planes de trabajos en procura de una formación integral del Licenciado. El taller se dividió en cuatro partes: (i) situación actual de la práctica profesional docente, (ii) análisis crítico del proceso de práctica profesional docente, (iii) alternativas de solución a la problemática de la práctica profesional docente y (iv) reflexiones generales. Las memorias fueron publicadas por el ICFES.
En 1984 se publica el trabajo Elementos Fundamentales del Perfil Teórico del Docente para el Departamento del Huila, llevado a cabo por los profesores Patricia Cristancho de Iriarte, Daniel Yovanovic, Miguel Angel Tovar y Antonio Iriarte C. La publicación se hizo a manera de folleto de 54 páginas y se le hizo la suficiente divulgación a nivel nacional. Este trabajo llevó a que dichos profesores publicaran igualmente en 1988 Los maestros del Huila: reconocimiento y transformación de su quehacer. El documento es prologado por el entonces decano, Jorge Elías Guebelly.

Durante mucho tiempo, el profesor Guebelly fue uno de los voceros de la Facultad de Educación en otros lugares del país. En 1987 precisamente presentó una ponencia titulada La Facultad de Educación de la Universidad Surcolombiana y la Crisis de la Educación en el Huila, en el marco de un evento preparado por el ICFES: La formación y capacitación del docente en Colombia. Las observaciones del profesor Guebelly aunque polémicas fueron muy útiles en su momento y nos cuestionan por nuestra situación actual como Facultad de Educación:

El plan curricular actual de la Facultad de Educación está superado por el desarrollo social del Huila y por el transcurrir del tiempo. No llena las expectativas de los huilenses. Responde con mediania y pobreza a las necesidades de la región. Esta realidad se extiende a la mayoría de planes curriculares existentes en el departamento. Existen las experiencias curriculares avanzadas pero son las excepciones. La educación en el Huila, incluyendo la de la facultad de Educación, está divorciada de su contexto; influye muy poco en el desarrollo integral del hombre huilense [...] La relación de los Programas de la Facultad de Educación con las diferentes instituciones educativas del Departamento son esporádicas. No obedecen a politicas interinstitucionales y están supeditadas, 
en la mayoría de los casos, a actividades particulares. En algunos casos se han firmado convenios que no se cumplieron o se cumplieron mal. En otros casos, los vasos comunicantes son débiles y se han hecho gracias a la voluntad de profesores y estudiantes. ${ }^{4}$

La década de los ochenta que cierra el profesor Lino Muñoz (periodo 1988-1991) dejó un buen número de proyectos de investigación ${ }^{5}$ para la universidad, siendo la Facultad de Educación un lider indiscutible:

1977: Atlas lingüistico del Huila. Primera parte, nivel léxico (monografia de grado). Recursos deportivos del Huila (práctica académica).

1979: Análisis ocupacional de Administrador Educativo (proyecto de facultad).

Causas sociales de los problemas de salud en Rivera (proyecto personal).

Asistencia técnico deportiva a las Juntas Municipales de Deportes (Trabajo interinstitucional).

Programa de integración universitaria para la docencia y la investigación del español americano (proyecto personal).

1980: Análisis cardio-pulmonar de los deportistas preseleccionados a los XI Juegos Nacionales en Neiva (monografia de grado).

La deserción universitaria en el programa de Preescolar (proyecto de facultad).

1981: Financiamiento de los clubes deportivos de las empresas oficiales de Neiva (monografia de gado).

1982: Diagnóstico de los centros docentes urbanos oficiales del nivel básico primario en el departamento del Huila en 1982 (práctica académica).

La práctica de la administración educacional en Colombia (proyecto de facultad).

Balance parcial de la campaña de Alfabetización
"Simón Bolivar" en Neiva durante 1981 (práctica académica).

Modelo surcolombiano para la Universidad Abierta y a Distancia (proyecto de facultad).

1983: Diseño de programas funcionales de Educación Fisica para la escuela primaria (proyecto de facultad).

Perfil profesional del docente en el Huila (proyecto de facultad).

Determinación de las caracteristicas profesionales del profesor universitario responsable del proceso de formación de licenciados en Educación Física (práctica académica).

Estado actual de la enseñanza de la fisica en el nivel medio oficial y privado del Huila (tesis de grado).

Seguimiento al egresado de Educación preescolar (proyecto de facultad).

Lineamientos para la capacitación del maestro de primero de primaria.

Grado de aprendizaje de los niños de primero de primaria, egresados del Jardin Universidad Surcolombiana (práctica académica).

Mejoramiento de la docencia universitaria (proyecto interdisciplinario).

1984: Campo ocupacional del licenciado en Educación Física: sus programas y sus recursos. Diseño de programas funcionales de educación física para la escuela primaria (proyecto de facultad).

Evaluación de la práctica profesional del programa de Administración Educativa: 1976 a 1983 (práctica académica).

Intereses cognitivo-comunicativos de los estudiantes de bachillerato de la ciudad de Neiva, sobre temas de valor universal (práctica académica).

Reestructuración del programa de Administración educativa de la Universidad surcolombiana (proyecto de facultad).

Guia administrativa para los centros docentes del nivel de básica (práctica académica).

1986: Manual de funciones para la organización 
y conservación de archivos municipales (proyecto de facultad).

1987: Construcción de un horno solar para secado de café (proyecto de facultad).

Diseño de paquetes instruccionales para la enseñanza de la fisica (proyecto de facultad).

Propuesta para la estructuración del sistema de investigación en la Universidad Surcolombiana (institucional).

1988: Análisis de la situación actual de la enseñanza del inglés y del francés en la educación básica secundaria y media vocacional en el departamento del Huila (proyecto de facultad).

Diseño y aplicación de un modelo de seguimiento a egresados (proyecto de facultad).

1989: El pensamiento de Vargas Llosa "Ensayo" (proyecto de facultad).

Prevención y orientación de las dificultades de aprendizaje en el nivel de básica primaria (proyecto de facultad).

1990: Rivera intelectual. Antologia de textos y documentos de José Eustasio Rivera (1912-1928) (proyecto de facultad).

Orientación de la enseñanza de la lengua materna al desarrollo de la competencia comunicativa en la educación básica.

Análisis de la Educación Fisica a nivel preescolar en el Huila: Implantación e implementación de un modelo curricular con orientación participativa (proyecto de facultad).

Evaluación diagnóstica comparativa de las condiciones técnico-administrativas y pedagógicas de la Educación Física en los preescolares del departamento del Huila (proyecto interfacultad).

Teniendo en cuenta las investigaciones citadas, la Facultad de Educaciones centró en cuatro tópicos de trabajo, cada uno con sus respectivas lineas, a saber:

1. Administración Educativa: Evaluación institucional y Educación para el desarrollo regional.

2. Pedagogia: Calidad de la educación, docencia universitaria y enseñanza de la ciencia.

3. Currículo: Diseño curricular y Evolución curricular.

4. Sistema educativo: Universidad y necesidades regionales.

En el periodo comprendido entre 1991 y 1995 (decanatura del profesor Fabio Losada), se inicia con el reto de la organización y realización del VIII Encuentro de Facultades de Educación del suroccidente colombiano que tuvo lugar los dias 29,30 y 31 de marzo de 1991 en la USCO y al cual asistieron cerca de cien representantes de Facultades de Educación y de instituciones formadoras de maestros. En este encuentro, el profesor Guebelly volvió a hacer presencia presentando una charla titulada Valores culturales y educación que luego se difundiria como separata de catorce páginas. En ese aporte, queda claro que la educación está en crisis y todo porque los maestros han caído en la mecanización de su oficio, han perdido la creatividad y se han olvidado de que su propia experiencia es tan válida como la de cualquier modelo extranjero. En realidad, la culpa no es de la abstracción, 'Facultades de Educación' sino de los seres reales, de carne y hueso cuyo trabajo es deficiente. Y el profesor Guebelly parece encontrar el malestar de la educación: la actitud tradicional de enseñar imponiendo valores culturales. Veamos lo que afirma: "Enseñar es hoy como ayer- hacer asimilar los modelos culturales del educador. Así, la enseñanza no es un diálogo para descubrir el sentido de la vida sino un monólogo para imponer una visión particular del mundo. Si el profesor es comunista o cristiano o conservador y tantas posibilidades más, sólo pretenden perpetuar sus valores axiológicos. Y una formación de 
imposiciones, no es libre, no libertaria, produce rechazo porque es muerte humana y sólo es posible realizarse mecánicamente. (págs. 9-10) La Facultad de Educación orientó la elaboración de un Plan de Desarrollo, a partir de un proceso participativo y que tenía como fin propiciar propuestas curriculares y un modelo pedagógico que respondiera a las exigencias actuales de la realidad regional y nacional. La Decanatura consideró la necesidad de crear cuatro comités de apoyo para llevar a cabo los compromisos institucionales, regionales asi como nacionales: Comité de Investigación, Comité de Extensión, Comité de Práctica Docente y Comité de Desarrollo Institucional.

Se buscaba con afán lograr la calidad de la educación en donde todos los miembros de la comunidad educativa participaran. El profesor Losada acertaba al afirmar que la calidad no se decreta sino que se busca en acciones propias por parte del maestro. Y la calidad en la formación de los maestros, se podía analizar desde tres perspectivas: (i) la de la excelencia académica de los programas que forman maestros, (ii) la de las instituciones encargadas de desarrollar dichos programas que está relacionada con las condiciones de racionalidad y de eficiencia organizacional para el logro de la excelencia académica y (iii) la del nivel de profesionalización y dignificación de la actividad docente que debe estar garantizada por el Estado, teniendo en cuenta que el maestro es un trabajador de la cultura, un intelectual, al que se le deben garantizar las condiciones para sumir su trabajo como profesional. ${ }^{6}$

La gestión académica administrativa dejó los siguientes resultados:

1. El apoyo económico de las administraciones anteriores de la Universidad, para acondicionar las instalaciones y mencionar el ambiente físico de trabajo.
2. Se revivió la edición de la revista de la facultad PAIDEIA.

3. Se avanzó en la implementación de la informática en la docencia requerida por la mayoría de los programas con el apoyo económico representativo del seño contralor Jaime Losada que luego fue complementado por el ICFES y la propia USCO para adquirir los computadores y crear el laboratorio de informática de la Facultad.

4. Se gestionó ante el ICFES la licencia de funcionamiento del programa de lenguas modernas negada en ocasiones anteriores. De igual forma comenzó a funcionar la especialización para la enseñanza de la biología en convenio con la Universidad del Tolima y la Maestría en Educación y Desarrollo Comunitario en convenio con el CINDE.

5. Se gestionó ante el ICFES la creación de la biblioteca especializada para los postgrados y se obtuvo un aporte de 4 millones de pesos.

Nace de la Facultad de Educación, en cabeza del profesor William Torres el pregrado en Comunicación social y procesos culturales con énfasis en radio y televisión, cumpliendo con todos los trámites e iniciando labores en el primer semestre de 1995.

Mediante el Acuerdo 0038 del 22 de Noviembre de 1994, el Consejo de Facultad de Educación creó la comisión de reestructuración de la Facultad, integrada por los profesores Aura Elena Bernal, Nelson López, Luis Evelio Vanegas, Jorge Elias Guebelly, Lino Muñoz y Miguel Angel Tovar para que redactaran un documento académico administrativo. La comisión consideró oportuno retomar como referente teórico algunos documentos relacionados con el proceso objeto de su función y fue asi como responsabilizó a cada uno de sus 
miembros del análisis y presentación de las siguientes elaboraciones documentales:

- Implicaciones de la Ley 115 de 1994 y su decreto reglamentario 1860 frente a las Facultades de Educación.

- Propuesta de reforma de las Normales y de la Facultad de Educación de la USCO.

- La Escuela, el Maestro y su Formación.

- Propuesta de reestructuración académico/ administrativa de la USCO.

- Informe de la Misión de Ciencia, Educación y Desarrollo.

- El proceso de Acreditación Universitaria.

El profesor Juan Manuel Perea llama la atención para que dicha reestructuración no se quede en el papel sino que logre su propósito cumpliendo con los siguientes objetivos:

- Responder a los retos científicos y tecnológicos del momento con el diseño y actualización de curriculos que propendan por el desarrollo de la creatividad en sus estudiantes, futuros educadores de la juventud.

- Responder a la sociedad con un perfil de educador que no sólo posea buenos conocimientos sino que además sea capaz de desarrollar nuevas ideas, nuevos conocimientos, para adaptarse a un mundo cambiante siempre nuevo e insospechado; es decir, gestor de nuevos procesos educativos y no simple repetidor.

- Adecuar y captar los recursos necesarios (salas de informática, biblioteca, laboratorios, ayudas didácticas, etc.) para impartir una formación menos teórica y más práctica con aplicaciones y soluciones al entorno.
En el año de 1996, el Consejo de Facultad de Educación llevó a cabo la estructuración de un plan básico de acción. En este plan se evidencian de manera integral, los componentes: administrativo, de investigación, de extensión y desde los programas académicos con el fin de plantear metas y acciones válidas para el desarrollo de la facultad.

La perspectiva histórica de la Facultad relaciona los periodos de desarrollo pasados con la situación presente y prospectiva, como bases para valorar la pertinencia y viabilidad de los programas en un futuro determinado, en este caso, con la temporalidad del Plan de Desarrollo. 2.004 - 2.010.

De acuerdo con la misión el propósito básico de la Facultad es egresar educadores profesionales formados integralmente como personas, ciudadanos y profesionales de la educación para su constante superación y el cambio sociocultural que requiere nuestro pais, como unidad académico-administrativa formadora de este tipo de educadores profesionales, debe liderar al interior de la Universidad un modelo pedagógico coherente y consistente con este propósito básico de formación. En palabras del entonces Decano, profesor Liberio Salazar:

La facultad de Educación de nuestra Universidad Surcolombiana quiere centrar su atención en dos de los factores determinantes del estado del arte de la Educación Superior en Colombia, a saber: el desempeño de los profesores de las instituciones universitarias y la interrelación del ejercicio de las dos funciones fundamentales de ese educador, la investigación y la docencia, vale decir, la interdependencia (como que se retroalimentan mutuamente) entre el investigador, el productor de nuevos conocimientos y el orientador, el formador, el propiciador de la apropiación crítica y creativa del saber por parte del estudiante, interactuando en procesos transformadores de la misma 
realidad educativa, desde esa posibilidad de abordar la educación en su complejidad, como escenario de relación y comunicación entre los sujetos que intervienen en ella a través de la mediación del conocimiento.

Con esta finalidad se trabajó el Seminario Permanente sobre Enfoques Pedagógicos en Educación Superior, el cual aportó durante los dos últimos años, dos importantes logros: La realización del Primer Encuentro Internacional de Prácticas Pedagógicas (2002) y la publicación del libro Seminario Permanente sobre la problemática pedagógica en la Educación Superior (2002).

Con relación al primero, el evento se llevo a cabo en el Centro de Convenciones José Eustasio Rivera los dias 7 y 8 de noviembre. El lema: Por la construcción de una nueva cultura académica y profesional. Se trataron, a través de las diferentes ponencias temas propios de un nuevo siglo, un siglo de tecnologia y globalización y su influencia en el campo educativo; conceptos como los de transversalidad, interdisciplinariedad y transdisciplinariedad en educación, saberes y métodos en la universidad, prácticas pedagógicas, seminario investigativo, etc. En el número 10 de la revista Paideia se hizo una breve semblanza del evento y se publicaron dos ponencias centrales: Saberes y aprendizajes en la universidad a cargo del profesor Armando Zambrano y Las prácticas pedagógicas: un lugar cultural de resistencia, por el profesor Gerardo Andrés Perafán. De igual forma, se publicó la ponencia del profesor Wisberto Navarro, El seminario investigativo como práctica pedagógica.

El seminario permanente sobre la problemática pedagógica en la educación superior produjo un libro titulado Encuentros y des-encuentros con la pedagogia en la educación superior. Como lo comenta el profesor Nelson López, la temática abordada giró en torno a problemáticas relacionadas con las tendencias pedagógicas contemporáneas que permitieron el conocimiento, estudio, análisis y reflexión sistemática sobre tópicos directamente asociados con el constructivismo, las clases sociales y las prácticas pedagógicas, las inteligencias múltiples, los modelos de práctica pedagógica, sobre las alternativas pedagógicas, la de-construcción curricular. El pensamiento docente y la práctica pedagógica.

El Diplomado en Docencia Universitaria, dirigido por la Coordinación de Extensión, ofrece formación en Pedagogia a los profesores de la Universidad; el Departamento de Psicopedagogia, oferta las cátedras en Investigación Formativa; el Departamento de Idiomas ofrece la enseñanza del Inglés y de otros idiomas; el Programa de Educación Fisica, Recreación y Deporte desarrolla las cátedras de Deporte Formativo y diferentes disciplinas deportivas y recreativas.

Por otro lado, es competencia de la Facultad, adelantar el debate sobre el sentido de la formación integral, rasgo incluido en la Misión de la Universidad. Según el doctor Alejandro Acosta (1996), el concepto de integralidad en la formación asume este proceso no solamente como una suma de componentes muy pertinentes, sino también, el pensar a los estudiantes y a los grupos académicos, como gestores de su propia formación.

En el ámbito académico institucional la Facultad de Educación mantuvo el liderazgo en la generación, diseño, adopción y ejecución de nuevos programas académicos de formación profesional a nivel de pregrado y postgrado: Inició con programas presenciales en jornada diurna y nocturna; después, adoptó la metodologia a distancia; posteriormente, abrió las maestrias mediante convenios con otras universidades y por último, logró la apertura de especializaciones propias. 
La investigación pedagógica sobre los procesos académicos de la universidad no ha tenido el apoyo deseable de la Universidad y debe ser un objetivo a lograr en el corto plazo.

A la Facultad le compete, también, investigar en los campos disciplinares especificos de cada uno de sus programas y esto se ha dado. El problema está en el predominio del énfasis de la investigación disciplinar sobre la investigación pedagógica.

En el campo del desarrollo institucional, la facultad se ha vinculado, a través de sus profesores, a los cargos y organismos de dirección, ejecución, coordinación, asesoría y a equipos de trabajo institucionales; ha aportado importantes ingresos financieros provenientes del porcentaje que le corresponde a la universidad, por el ofrecimiento de programas propios, mediante convenios suscritos con otras universidades y por proyectos interinstitucionales. Sin embargo, el apoyo financiero y administrativo por parte de la universidad, no ha sido el deseable, en cuanto se refiere a mantener la planta de los docentes, mejorar la dotación bibliográfica, las aulas y oficinas y la dotación de equipos y elementos de apoyo didáctico.

Según el informe que la profesora Ligia Lavao de Serrato, actual Decana de la facultad presentó ante la Asamblea Departamental, en la actualidad la Facultad de Educación está conformada de la siguiente forma:

\section{Programas}

1.Programa de Licenciatura en Ciencias Naturales y Educación Ambiental

Aprobado mediante Resolución No. 094, del 27 de enero de 2000 , la acreditación previa, en la sede de Neiva, con metodologia presencial, jornada diurna y fue incorporado al Sistema
Nacional de Información de la Educación Superior (SNIES), el 4 de octubre de 2002, con el código 111443705004100111100.

2. Programa de Licenciatura en Educación Física, Recreación y Deportes

Fue creado por Acuerdo 014 del 17 de octubre de 1975, del Consejo Superior Universitario. El ICFES le concedió licencia de iniciación de labores por Acuerdo 191 de diciembre 7 de 1977. Con Acreditación de Alta Calidad otorgada mediante Resolución No 3769, del 5 de julio de 2007 y Registro calificado ICFES No 111446130704100111100 de 22 de septiembre de 2000.

Su duración es de ocho semestres académicos, según Plan de estudios aprobado, según Acuerdo No 107 del 7 de diciembre de 2004 del Consejo de Facultad de Educación, en jornada diurna.

3. Programa de Licenciatura en Educación Artistica

Obtuvo registro calificado mediante Resolución 094 del 27 de enero de 2000, con metodología presencial jornada diurna, incorporado al Sistema Nacional de Educación superior SNIES con Código 11144372700410011110.

4. Programa de Licenciatura en Matemáticas Se creó por Acuerdo 013 de octubre 17 de 1975, emanada del Consejo Superior de la Universidad Surcolombiana. La aprobación le fue otorgada por Acuerdo 096 de 1980.

El 27 de enero del año 2000, el Ministerio de Educación Nacional expide la Resolución No. 094 por la que otorga Acreditación Previa a algunos programas, entre los que está la Licenciatura en Matemáticas y se ordena al ICFES su inscripción en el SNIES, hecha el 4 de octubre de 2000, con el código 111445103704100111100 , en la modalidad presencial, con jornada diurna y una duración de 10 semestres académicos. 
5. Programa de Licenciatura en Lengua Castellana

Fue creada por Acuerdo 066 del 14 de mayo de 1973 como Tecnología de Español y Literatura. En el año de 1976 se le otorga licencia de funcionamiento al programa como Licenciatura en lingüistica y Literatura.

Obtuvo registro calificado mediante Resolución 094 del 27 de enero de 2000, con metodología presencial jornada diurna, incorporado al Sistema Nacional de Educación superior SNIES con Código 11143724004100111101.

6. Programa de Licenciatura en Lengua Extranjera - Inglés

El hoy denominado Programa de Licenciatura en Educación Básica con énfasis en Humanidades, Lengua Extranjera - Inglés, consta de 150 créditos académicos, en jornada diurna, modalidad presencial y registrada ante el SNIES bajo el código 111443724004100111101.

Hoy, el Programa tiene Acreditación de Alta Calidad, otorgada mediante Resolución No. 3575 , del 16 de junio de 2008, emanada del Ministerio de Educación Nacional.

7. Programa de Licenciatura en Pedagogia Infantil.

Inició en la modalidad de tecnologia mediante Acuerdo 94 de 1974 emanada del ICFES. En noviembre de 1975 por Acuerdo 241, el ICFES le concede aprobación.

Desde 1998, el Programa se denomina Licenciatura en Pedagogia Infantil y se interesa en formar profesionales con dominio en el campo pedagógico específico, investigativo y de proyección a las comunidades vulnerables. Su actual plan de estudios está estructurado para ser desarrollado en 8 semestres académicos.

Con registro calificado mediante Resolución 094 del 27 de enero de 2000, metodologia presencial jornada diurna, incorporado al Sistema
Nacional de Educación superior SNIES con Código 111443720014100111100.

\section{Características académico-administrativas}

\section{Teleologia}

Por su innegable naturaleza pedagógica la Facultad debe ser vista desde la perspectiva de su Misión que se expresa de la siguiente manera: "La Facultad de Educación de la Universidad Surcolombiana tiene como misión la formación de educadores con un sentido humanista e integral, en las diferentes áreas del conocimiento y la producción y consolidación de nuevos saberes y en lo esencial el pedagógico, para el mejoramiento de la educación como factor fundamental del desarrollo integral, equitativo y sostenible de la región surcolombiana, el pais y la esfera internacional".

De esto se desprende el carácter humanista que rige los actos de la Facultad, es decir, su preocupación por la formación de un ser humano que responda a la generación de saberes articulados con las expectativas y necesidades de la región y el pais y con otras disciplinas que alimentan el quehacer pedagógico y orientan la labor didáctica.

Es por ello que la Facultad pretende, con un horizonte del 2010, que ya debe ser replanteado en el tiempo, ejercer un liderazgo efectivo, más no sólo en la formación de una masa profesional critica, sino de una persona cuya excelencia debe expresarse en sus actos como pedagogo y como ser humano. Textualmente, la Visión reza que:

"En el año 2010 la Facultad de Educación de la Universidad Surcolombiana será reconocida por el liderazgo profesional, pedagógico y humano de sus egresados y del personal que la integra. El liderazgo se basa en la excelencia académica y la administrativa, en la modernización de su infraestructura, en el desarrollo de la investigación y los postgrados". 
Desde esta óptica, sus propósitos de formación de educadores destacados en lo humano, lo científico, lo pedagógico, lo ético y lo político se deben orientar tanto a la dimensión individual, como colectiva, resaltando con ello el gran compromiso social que atraviesa todas las acciones que emprenda la comunidad académica de la Facultad de Educación. De aquí se deriva el primer propósito ("Formar, desde los proyectos académicos, educadores destacados por su calidad humana, cientifica, pedagógica, ética y política, que propendan por el desarrollo integral del ser humano y la sociedad"), enmarcado en un ambiente de diálogo democrático y participativo que ayude en la tarea nacional de construcción de paz, como se expresa en su segundo propósito: "Propiciar las condiciones legales, institucionales, orgánicas y operativas que favorezcan formación de educadores bajo la forma de relaciones dialógicas, para la vida democrática, participativa, para la convivencia armónica y para construir la paz nacional".

Metodológicamente, este propósito colectivo será alcanzado siempre y cuando se impulse el espiritu y la actividad investigativa bajo la mirada de diferentes miradas disciplinares en una dinámica de interacción permanente con la región, vista no como espacio geográfico, sino como categoria conceptual construida colectivamente y sometida a constante revisión.

Es por esto que sus objetivos están postulados de la siguiente manera:

- "Desarrollar estrategias de formación permanente que contribuyan al continuo mejoramiento de la calidad de la educación y a la evaluación del nivel intelectual y de la capacidad valorativa de los docentes para su realización personal, la cualificación en el desempeño profesional y en su proyección social.

- Evaluar la calidad, pertinencia e impacto social de las diferentes acciones de formación de educadores, investigación y desarrollo social que le permitan a la Facultad posicionarse como agente dinamizador del sistema educativo regional.

- Potenciar el profesores y estudiantes las aptitudes y actitudes necesarias para la promoción educativa, la investigación tanto en el campo pedagógico como en los saberes especificos y el desarrollo de procesos de procesos integrados de docencia, investigación, extensión, gestión educativa y aplicación de nuevas tecnologias".

Estos planteamientos llevan, obligatoriamente, a considerar la pertinencia social y politica de la Facultad en una región afectada de primera mano por los conflictos sociales. Es asi como el Plan de Desarrollo se ha titulado "Educación con responsabilidad social", denotando el interés colectivo por el ser humano y la región, dentro de un contexto universal.

Asi mismo, uno de los propósitos centrales de la Facultad gira en torno a la necesidad de construir relaciones de diálogo permanente como base de la vida democrática, participativa y armónica. Es por ello, que desde el campo de la educación formal, la Facultad ofrece los proyectos académicos de titulación en la áreas de la cultura fisica, el estudio de la lengua nacional y extranjera, la exploración de las formas esenciales de vida, el necesario desarrollo de las dimensiones propias de la niñez y el avance en el pensamiento racional y emocional.

Pero también desde lo no formal, la Facultad de Educación implementa propuestas en derechos humanos, en atención poblaciones con necesidades educativas especiales, en sistemas alternativos de comunicación humana, en docencia universitaria, etc.

Cifras

Hoy, la Facultad cuenta con más de 2000 estudiantes de estratos populares como se puede apreciar en los Cuadros 
FACULTAD DE EDUCACIÓN

ESTRATIFICACIÓN Y ORIGEN ESTUDIANTES MATRICULADOS SEMESTRE A/2009

\begin{tabular}{|c|c|c|c|c|c|c|c|}
\hline \multirow{2}{*}{ NOMBRE PROGRAMA } & \multirow{2}{*}{ N" ESTUDIANTES } & \multicolumn{4}{|c|}{ ESTRATO } & \multicolumn{2}{|c|}{ INSTITUCION } \\
\hline & & 1 & 2 & $\mathbf{3}$ & 4 & OFICLAL & PRIVADA \\
\hline $\begin{array}{l}\text { Lic. Ed. Básica énfasis } \\
\text { Ciencias Naturales y } \\
\text { Educación Ambiental }\end{array}$ & 42 & 11 & 29 & 2 & 0 & 29 & 13 \\
\hline $\begin{array}{c}\text { Lic. Ed. Básica énfasis en } \\
\text { Humanidades Lengua } \\
\text { Castellana }\end{array}$ & 31 & 13 & 18 & 0 & 0 & 29 & 2 \\
\hline $\begin{array}{c}\text { Lic. Ed. Básica énfasis en } \\
\text { Humanidades Lengua } \\
\text { Extranjera }\end{array}$ & 40 & 14 & 22 & 2 & 2 & 32 & 8 \\
\hline $\begin{array}{l}\text { Lic. Ed. Básica énfasis en } \\
\text { Educación Artística }\end{array}$ & 39 & 18 & 19 & 2 & 0 & 28 & 11 \\
\hline $\begin{array}{l}\text { Lic. Ed. Básica énfasis en } \\
\text { Educación Física, } \\
\text { Recreación y D. }\end{array}$ & 38 & 12 & 25 & 1 & 0 & 28 & 10 \\
\hline Lic. en Matemáticas & 46 & 14 & 30 & 2 & 0 & 35 & 11 \\
\hline Lic. Pedagogía Infantil & 43 & 10 & 32 & 1 & 0 & 31 & 9 \\
\hline TOTAL & $279 / 1(0) \%$ & $92 / 33 \%$ & $175 / 63 \%$ & $10 / 3.5 \%$ & $2 / 0.5 \%$ & $215 / 77 \%$ & $64 / 23 \%$ \\
\hline
\end{tabular}

\section{A manera de conclusión}

La Facultad de Educación ha propuesto en su Plan de Desarrollo 2009-2013, lograr niveles de excelencia a partir de tres ejes: (i) Docencia, (ii) Investigación/Proyección Social y (iii) Gestión Académica/administrativa. Paralelo a este trabajo, nos parece que el desarrollo de dichos ejes, por el 'método de problemas', ayudaria a dimensionar adecuadamente los trabajos ya realizados sobre los cuales se desarrollarian las nuevas tareas y permitiria la consolidación del Proyecto Pedagógico de la Facultad.

\section{Bibliografía}

Patricia Cristancho de Iriarte et al. 1985. Elementos Fundamentales del Perfil Teórico del Docente para el Departamento del Huila. Neiva, Universidad Surcolombiana.

Jorge Elias Guebelly. 1987. La Facultad de
Educación de la Universidad Surcolombiana y la crisis de la Universidad en el Huila, en La Formación y Capacitación del Docente en Colombia. Memorias. Bogotá D.E. ICFES, pp. 53-60.

Patricia Cristancho de Iriarte et al. 1987. Los Maestros del Huila: Reconocimiento y Transformación de su Quehacer. Neiva, Cuaderno Surcolombiano 11.

Mankeliunas, M. (1993). Desarrollo histórico, en Psicología en Colombia. Contexto social e histórico, (pp. 43-66). Bogotá, TM Editores.

Revista Paideia Surcolombiana 10, agostodiciembre 2002.

Informe a la Asamblea Departamental. Facultad de Educación, Universidad Surcolombiana, marzo de 2009. 